\title{
Numerical spectral synthesis of breather gas for the focusing nonlinear Schrödinger equation
}

\author{
Giacomo Roberti, ${ }^{1}$ Gennady El, ${ }^{1}$ Alexander Tovbis, ${ }^{2}$ François Copie, ${ }^{3}$ Pierre Suret, ${ }^{3}$ and Stéphane Randoux ${ }^{3, *}$ \\ ${ }^{1}$ Department of Mathematics, Physics and Electrical Engineering, \\ Northumbria University, Newcastle upon Tyne, NE1 8ST, United Kingdom \\ ${ }^{2}$ Department of Mathematics, University of Central Florida, Orlando, USA \\ ${ }^{3}$ Univ. Lille, CNRS, UMR 8523 - PhLAM - Physique des Lasers Atomes et Molécules, F-59 000 Lille, France
}

\begin{abstract}
We numerically realize breather gas for the focusing nonlinear Schrödinger equation. This is done by building a random ensemble of $N \sim 50$ breathers via the Darboux transform recursive scheme in high precision arithmetics. Three types of breather gases are synthesized according to the three prototypical spectral configurations corresponding the Akhmediev, Kuznetsov-Ma and Peregrine breathers as elementary quasi-particles of the respective gases. The interaction properties of the constructed breather gases are investigated by propagating through them a 'trial' generic breather (Tajiri-Watanabe) and comparing the mean propagation velocity with the predictions of the recently developed spectral kinetic theory (El and Tovbis, PRE 2020).
\end{abstract}

\section{INTRODUCTION}

The study of nonlinear random waves in physical systems well described at leading order by the so-called integrable equations, such the Korteweg-de Vries (KdV) or nonlinear Schrödinger (NLS) equations has recently become the topic of intense research in several areas of nonlinear physics, notably in oceanography and nonlinear optics. This interest is motivated by the complexity of many natural or experimentally observed nonlinear wave phenomena often requiring a statistical description even though the underlying physical model is, in principle, amenable to the well-established mathematical techniques of integrable systems theory such as inverse scattering transform (IST) or finite-gap theory (FGT) [1]. An intriguing interplay between integrability and randomness in such systems is nowadays associated with the concept of integrable turbulence introduced by V. Zakharov in [2]. The integrable turbulence framework is particularly pertinent to the description of modulationally unstable systems whose solutions, under the effect of random noise, can exhibit highly complex spatiotemporal dynamics that are adequately described in terms of turbulence theory concepts, such as the distribution functions, ensemble averages, correlations etc.

Solitons and breathers are the elementary "quasiparticles" of nonlinear wave fields in integrable systems which can form ordered coherent structures such as modulated soliton trains and dispersive shock waves $[3,4]$, "superregular breathers" $[5,6]$ or "breather molecules" [7]. Furthermore, solitons and breathers can form irregular structures or statistical ensembles that can be viewed as soliton and breather gases. The nonlinear wavefield in such integrable gases represents a particular case of integrable turbulence [2, 8-13]. The observations of soliton and breather gases in the ocean have been reported in

*stephane.randoux@univ-lille.fr
[14-16]. Recent laboratory experiments on the generation of shallow-water and deep water soliton gases were reported in [17] and [18] respectively. It has also been demonstrated that the soliton gas dynamics in the focusing NLS equation provides a remarkably good description of the statistical properties of the nonlinear stage of spontaneous modulational instability [19].

Analytical description of soliton gases was initiated by Zakharov in ref. [20], where a spectral kinetic equation for KdV solitons was derived using an IST based phenomenological procedure of computing an effective adjustment to a soliton's velocity in a rarefied gas due to its collisions with other solitons, accompanied by appropriate phase-shifts. Zakharov's kinetic equation for KdV soliton has been generalized to the case of a dense gas in ref [21] using the spectral finite-gap theory. Within this theory, a uniform (equilibrium) soliton gas is modelled by a special infinite-phase, thermodynamic type limit of finite-gap KdV solutions. The kinetic description of the non-equilibrium soliton gas is then enabled by considering the same thermodynamic limit for the associated modulation (Whitham) equations. The resulting kinetic equation describes the evolution of the density of states (DOS) defined as the density function in the spectral (IST) phase plane of soliton gas. The spectral construction of the KdV soliton gas in ref. [21] has been generalized to the soliton gas of the focusing NLS equation in $[22,23]$. The latter work [23] provides also the spectral kinetic description of a breather gas (BG), which is the main subject of the present work.

An isolated generic breather can be broadly viewed as a soliton on the plane wave (or finite) background. The 1D-NLSE equation supports a large family of breather solutions that have attracted a particular interest due to their explicit analytic nature and the potential for modeling the rogue wave events in the ocean and in nonlinear optical fibers [24-28]. Three types of breathers, namely the Akhmediev breather (AB), the Kuznetsov$\mathrm{Ma}$ (KM) breather and the Peregrine soliton (PS) have aroused significant research interest, see [29-34] and ref- 
erences therein. AB, KM breather and PS represent special cases of a generic breather called the Tajiri-Watanabe (TW) breather [35]. A simplest example of breather gas can be viewed as an infinite random ensemble of the TW breathers [23]. By manipulating the spectral parameters the TW breather gas can be reduced to the AM, KM and PS gases as well as to the fundamental soliton gas. The latter is achieved by vanishing the plane wave background of the TW breather gas [23].

The present paper has two goals: (i) numerical realization of a breather gas; (ii) verification of the spectral theory of breather gas developed in [23].

Numerical realization of a breather gas as a large ensemble of TW breathers with prescribed parameters represents a challenging problem. Numerical methods for the construction of breather solutions of the 1D-NLSE suffer from accuracy problems that prevent the numerical synthesis of breathers of order $N \gtrsim 5[36,37]$. In the context of soliton gases this latter difficulty has been recently resolved by Gelash and Agafontsev [38] via the application of the so-called dressing method combined with high precision numerical computations. In this paper, we extend the algorithm of [38] to numerically realize various breather gases and verify some predictions of the spectral kinetic theory of [23]. In particular we demonstrate that random ensembles of $N \sim 50$ breathers can be build via the Darboux transform recursive scheme in high precision arithmetics. To our knowledge, this represents an improvement of an order of magnitude compared to the results reported in previous numerical works. In addition we show that the construction method can be used to provide evidence of the space-time evolution of the generated breather gases. This feature cannot be achieved by using direct numerical simulations of the 1D-NLSE due to the inevitable presence of modulational instability that quickly desintegrates the plane wave background.

The paper is organized as follows. In Section II we present the algorithm of the spectral synthesis of breather gas using the Darboux transform. This algorithm is then realized numerically using the high precision arithmetics. In Section III we numerically study the interactions in breather gases and compare the results of the numerical simulations with the theoretical predictions of the breather gas kinetic theory of Ref [23]. Specifically, we consider the propagation of the 'trial' breather through a homogeneous breather gas for three prototypical configurations: Akhmediev, Kuznetsov-Ma and Peregrine gases. The study of interaction in the gas of Akhmediev breathers has revealed some special features that have required further development of the theory of Ref [23]. The Appendix provides the identification of the interaction kernel in the breather gas with the position shift formula in two-breather collisions, obtained in earlier works.

\section{NONLINEAR SPECTRAL SYNTHESIS OF BREATHER GASES}

\section{A. Soliton and breather ensembles in the 1D-NLSE: an overview}

We consider the integrable one-dimensional focusing NLS equation (1D-NLSE) in the following form:

$$
i \psi_{t}+\psi_{x x}+2|\psi|^{2} \psi=0
$$

where $\psi(x, t)$ represents the complex envelope of the wave field that evolves in space $x$ and time $t$.

In the inverse scattering transform (IST) method, the 1D-NLSE (1) is represented as a compatibility condition of two linear equations [1, 39],

$$
\begin{gathered}
\Phi_{x}=\left(\begin{array}{cc}
-i \lambda & \psi \\
-\psi^{*} & i \lambda
\end{array}\right) \Phi \\
\Phi_{t}=\left(\begin{array}{cc}
-2 i \lambda^{2}+i|\psi|^{2} & i \psi_{x}+2 \lambda \psi \\
i \psi_{x}^{*}-2 \lambda \psi^{*} & 2 i \lambda^{2}-i|\psi|^{2}
\end{array}\right) \Phi,
\end{gathered}
$$

where $\lambda$ is a (time-independent) complex spectral parameter and $\Phi(x, t, \lambda)=(r(x, t, \lambda), s(x, t, \lambda))^{T}$ is a column vector. The spatial linear operator (2) and the temporal linear operator (3) form the Lax pair of Eq. (1). For a given potential $\psi(x, t)$ the problem of finding the scattering data $\sigma[\psi]$ (also sometimes called the IST spectrum) and the corresponding scattering solution $\Phi$ specified by the spatial equation (2) is called the Zakharov-Shabat (ZS) scattering problem [40]. The ZS scattering problem is formally analogous to calculating the Fourier coefficients in Fourier theory of linear systems, hence the term 'Nonlinear Fourier Transform' is often used in the context of telecommunications systems research, particularly in the context of periodic boundary conditions [41-43].

For spatially localized potentials $\psi$ such that $\psi(x, t) \rightarrow$ 0 as $|x| \rightarrow \infty$, the complex eigenvalues $\lambda$ are generally presented by a finite number of discrete points with $\Im(\lambda) \neq 0$ (discrete spectrum) and the real line $\lambda \in \mathbb{R}$ (continuous spectrum). The scattering data $\sigma(\psi)$ consist of a set of $N$ discrete eigenvalues $\lambda_{n}(n=1, \ldots, N)$, a set of $N$ norming constants $C_{n}$ for each $\lambda_{n}$ and the so-called reflection coefficient $\rho(\xi)$,

$$
\sigma(\psi)=\left\{\rho(\xi) ; \lambda_{n}, C_{n}\right\}
$$

where $\xi \in \mathbb{R}$ denotes the continuous spectrum component. In this setting where the wavefield $\psi$ lives on a zero background (ZBG), the discrete part of the IST spectrum is related to the soliton content of the wavefield whereas the continuous part of the IST spectrum is related to the nonlinear dispersive radiation [40].

A special class of (reflectionless) solutions of Eq. (1), the $N$-soliton solutions (N-SS's), exhibits only a discrete spectrum $(\rho(\xi)=0)$ consisting of $N$ complex-valued eigenvalues $\lambda_{n}, n=1, \ldots, N$ and $N$ associated complexvalued norming constants. The IST formalism has been 
extensively applied to examine the processes of interaction, collision and synchronisation in N-SS's, see e.g. ref. $[40,44]$. The numerical synthesis of N-SS's can be achieved in standard computer simulations (double precision, 16-digits) up to $N \sim 10$ [38]. On the other hand the numerical synthesis of N-SS's with $N$ large represents a challenging problem that has been resolved only recently [38]. Combining the so-called dressing method and numerical calculations made using high numerical precision (a 100-digits precision is typically necessary for the synthesis of N-SS's with $N \sim 100$ ), the numerical synthesis of soliton gases (SGs), i. e. large ensembles of N-SS's characterized by a given spectral distribution, has been demonstrated in ref. [38]. The opportunity to synthesize numerically large soliton ensembles has opened the way to the experimental generation of strongly nonlinear wavefields with a pure solitonic content. In particular recent experiments made in a one-dimensional water tank with deep-water surface gravity waves have revealed that the controlled synthesis of dense SGs can be achieved in hydrodynamics [18]. Moreover, it has been also recently shown that the so-called bound state SGs provide a model that describes well the nonlinear stage of the noise-induced modulation instability [19].

In addition to the soliton solutions living on zero background, the focusing NLS equation (1) admits a large variety of solutions living on a nonzero (plane wave) background. The IST theory for the focusing nonlinear Schrödinger equation with nonzero boundary conditions (NZBC) at infinity has been reported in ref. [45-47]. As in IST with zero boundary conditions, the scattering data $\sigma[\psi]$ in the IST with NZBC consist of a set of $N$ discrete complex-valued eigenvalues $\lambda_{n}$, a set of $N$ associated norming constants $C_{n}$ and the reflection coefficient $\rho(\lambda)$. In IST with NZBC, the continuous spectrum does not live on the real axis $\mathbb{R}$ but on $\mathbb{R} \cup\left[-i q_{0}, i q_{0}\right]$ where $q_{0}>0$ represents the amplitude of the plane wave background $[45,46]$.

The focusing NLS equation with NZBC possesses a rich family of purely solitonic solutions (reflectionless potentials, $\rho(\lambda)=0$ ) named breathers or sometimes solitons on finite background. The generic "elementary" breather parametrized by one single complex-valued eigenvalue $(N=1)$ in the framework of IST with NZBC is the so-called Tajiri-Watanabe breather [35]. This elementary solution reduces under certain limits to the solutions found over the years by Kuznetsov and Ma [29, 47], Peregrine [30], and Akhmediev [31]. Using the dressing method, Zakharov and Gelash constructed a class of two-soliton solutions on finite background, termed superregular breathers and corresponding to small initial perturbations of a constant background [48]. This was generalized to several pairs of breathers in ref. [5, 49]. Note that most of these breather solutions of Eq. (1) have been experimentally realized in hydrodynamics and in optics $[6,7,32,33,50-55]$.

\section{B. Darboux transform-based synthesis of breather gases}

The recent interest in studying the breather solutions of various kind has been fuelled by the rogue wave research, see e.g. [56] and references therein. The prototype rogue-wave solutions represent coherent structures of large amplitude, strongly localized in both space and time, on an otherwise quiescent background $[24,26,37,57-63]$. In this context the Darboux transform has been extensively used as a reliable method to generate higher-order breather solutions of Eq. (1), i.e. reflectionless solutions of the focusing 1D-NLSE with NZBC $[36,64-67]$. Note that the Darboux transform is now also used in the context of nonlinear eigenvalue communication to build ordered soliton ensembles used to carry out the transmission of information in fiber optics communication links [42, 43, 68].

The Darboux method is a recursive transformation scheme where a "seeding solution" of the focusing 1DNLSE is used as a building block for the construction of a higher-order solution through the addition of one discrete eigenvalue. Here we give a brief review of the Darboux transform method used for the generation of higher-order breathers. We largely follow the exposition given in ref. [37, 69] but other important references where this method is described and used are ref. [36, 64-67].

In the IST for the 1D-NLSE with NZBC, the seeding solution commonly used at the first step of the recursive process of constructing a higher-order breather solution is the plane wave solution of Eq. (1) with unit amplitude, i.e. $\psi_{0}(x, t)=e^{2 i t}$. The first-order breather (Tajiri-Watanabe) $\psi_{1}(x, t)$ parametrized by the complex eigenvalue $\lambda_{1}$ is obtained by

$$
\psi_{1}(x, t)=\psi_{0}(x, t)+\frac{2\left(\lambda_{1}^{*}-\lambda_{1}\right) s_{1,1} r_{11}^{*}}{\left|r_{1,1}\right|^{2}+\left|s_{1,1}\right|^{2}} .
$$

The functions $r_{1,1}(x, t)$ and $s_{1,1}(x, t)$ in Eq. (5) are obtained by setting $j=1$ in the following expressions

$$
\begin{gathered}
r_{1, j}(x, t)=2 i e^{-i t} \sin \left(A_{j}\right), \\
s_{1, j}(x, t)=2 e^{i t} \cos \left(B_{j}\right),
\end{gathered}
$$

where $A_{j}$ and $B_{j}$ are given by

$$
\begin{array}{r}
A_{j}=\frac{1}{2}\left(\arccos \left(\frac{\kappa_{j}}{2}\right)+\left(x-x_{j}\right) \kappa_{j}-\frac{\pi}{2}\right) \\
+\left(t-t_{j}\right) \kappa_{j} \lambda_{j}, \\
B_{j}=\frac{1}{2}\left(-\arccos \left(\frac{\kappa_{j}}{2}\right)+\left(x-x_{j}\right) \kappa_{j}-\frac{\pi}{2}\right) \\
+\left(t-t_{j}\right) \kappa_{j} \lambda_{j},
\end{array}
$$

with $\kappa_{j}=2 \sqrt{1+\lambda_{j}^{2}}$. The parameters $\left(x_{j}, t_{j}\right)$ are connected with the complex norming constants $C_{j}$ in the IST with NZBC [36]. The first-order breather $\psi_{1}(x, t)$ is parametrized by the complex eignevalue $\lambda_{1}$ and by the two real parameters $x_{1}$ and $t_{1}$. Once the first-order 

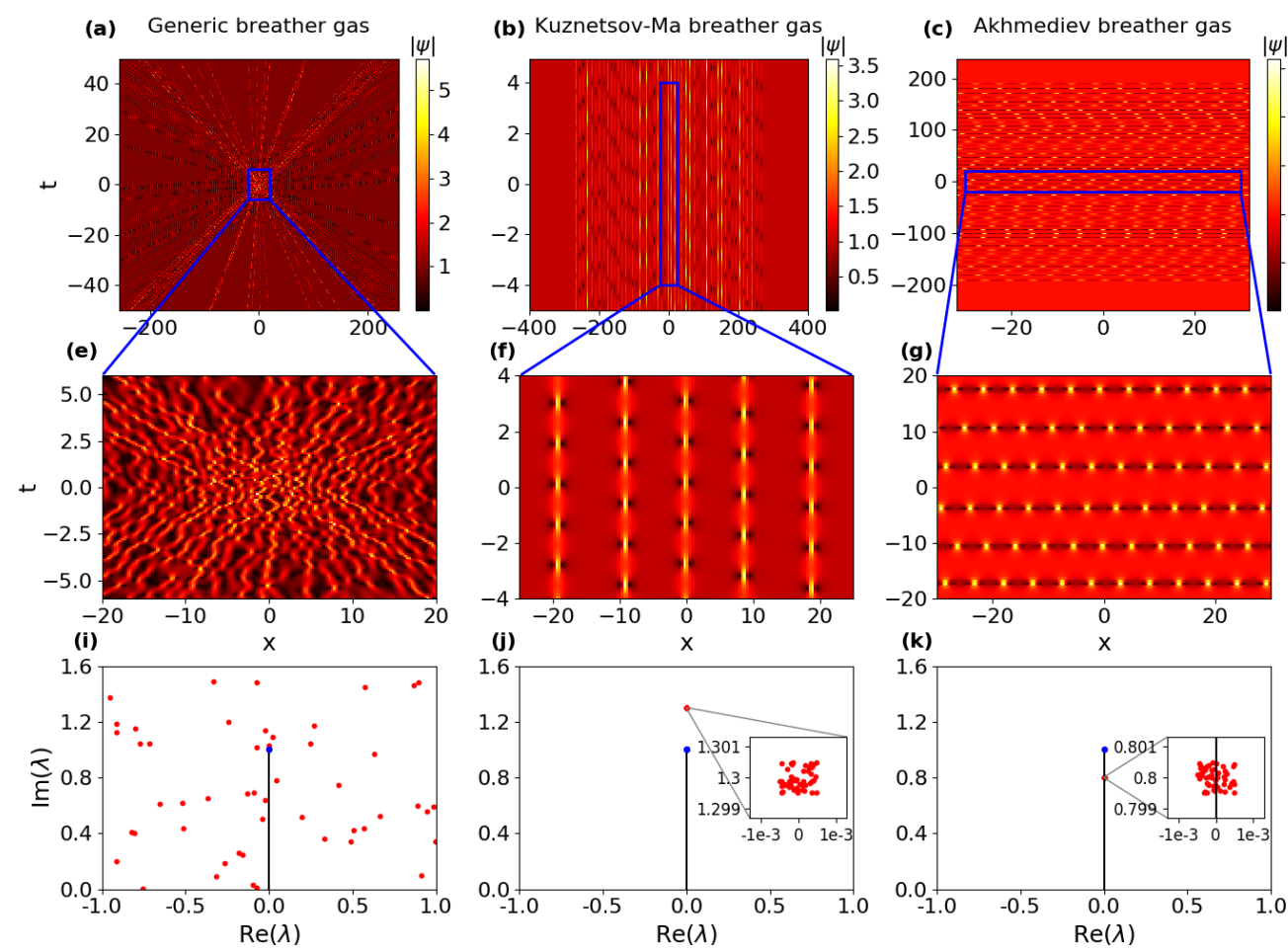

(d) Peregrine breather gas
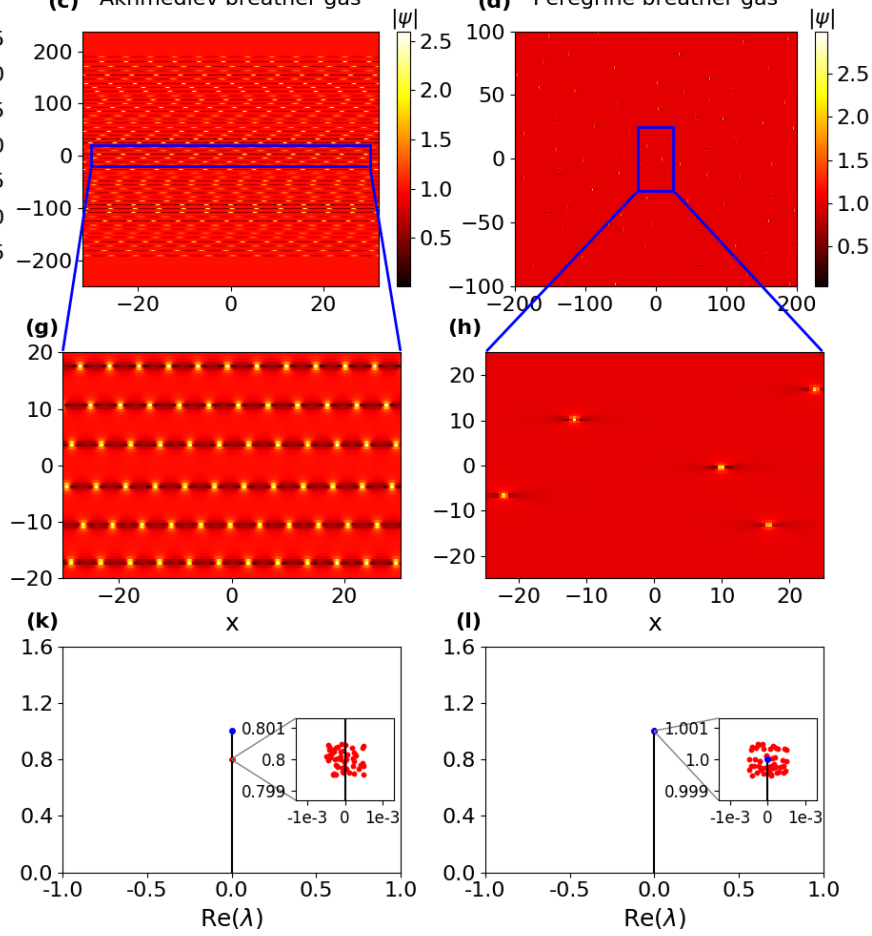

Figure 1. Numerical synthesis of a generic BG (left column (a), (e), (i)) and of three single-component BGs: a KM-BG (second column (b), (f), (j)), a AB-BG( third columnn (c), (g), (k)) and a PS-BG (fourth column (d), (h), (i)). The four BGs are parametrized by $N=50$ complex eigenvalues $\lambda_{n}$, see bottom row. The first row (a)-(d) represents the space-time evolution of the BGs, with the second row (e)-(h) being an enlarged view of some restricted region of the $(x-t)$ plane. The third row (i)-(l) represents the spectral portraits of each BG with the vertical line between 0 and $+i$ being the branchcut associated with the plane wave background. Each point in the upper complex plane in $(\mathrm{i}),(\mathrm{j}),(\mathrm{k}),(\mathrm{l})$ represents a discrete eigenvalue in the IST problem with NZBC. The eigenvalues parametrizing the single-component BGs are densely placed in a small square region which is centered around a point $\lambda_{0}$ of the imaginary vertical axis and which is strongly enlarged in the insets shown in ( $\mathrm{j}$ ), $(\mathrm{k}),(\mathrm{l})$. The $x_{j}$ are uniformly distributed in the range $[-1,1]$ for the generic gas (a) and for the Peregrine gas (d) while they are uniformly distributed in the range $[-32,32]$ for the KM gas (b) and the AB gas (c).

breather $\psi_{1}$ is constructed using Eqs. (5), (6), (7), breather solutions of order $n \geq 2$ can be recursively generated by using

$$
\psi_{n}(x, t)=\psi_{n-1}(x, t)+\frac{2\left(\lambda_{n}^{*}-\lambda_{n}\right) s_{n, 1} r_{n, 1}^{*}}{\left|r_{n, 1}\right|^{2}+\left|s_{n, 1}\right|^{2}}
$$

with

$$
\begin{array}{r}
r_{n, p}=\left[\left(\lambda_{n-1}^{*}-\lambda_{n-1}\right) s_{n-1,1}^{*} r_{n-1,1} s_{n-1, p+1}\right. \\
+\left(\lambda_{p+n-1}-\lambda_{n-1}\right)\left|r_{n-1,1}\right|^{2} r_{n-1, p+1} \\
\left.+\left(\lambda_{p+n-1}-\lambda_{n-1}^{*}\right)\left|s_{n-1,1}\right|^{2} r_{n-1, p+1}\right] / \\
\left(\left|r_{n-1,1}\right|^{2}+\left|s_{n-1,1}\right|^{2}\right), \\
s_{n, p}=\left[\left(\lambda_{n-1}^{*}-\lambda_{n-1}\right) s_{n-1,1} r_{n-1,1}^{*} r_{n-1, p+1}\right. \\
+\left(\lambda_{p+n-1}-\lambda_{n-1}\right)\left|s_{n-1,1}\right|^{2} s_{n-1, p+1} \\
\left.+\left(\lambda_{p+n-1}-\lambda_{n-1}^{*}\right)\left|r_{n-1,1}\right|^{2} s_{n-1, p+1}\right] / \\
\left(\left|r_{n-1,1}\right|^{2}+\left|s_{n-1,1}\right|^{2}\right) .
\end{array}
$$

Despite the efficiency of the Darboux method for the construction of high-order breather solutions of Eq. (1), its practical implementation in numerics suffers from the same type of issues as those previously mentioned for the numerical construction of N-SS's. As noted in ref. $[36,37]$, problems of numerical accuracy may prevent the numerical synthesis of breathers of order $N \gtrsim 5$. In this paper we show that this limit can be overcome by the implementation of the same strategy as the one used to build N-SS's with N large [38]. Implementing the Darboux recursive scheme in high precision arithmetics using the BOOTS $\mathrm{C}++$ Multiple precision Library, we show that breather solutions of Eq. (1) can be synthesized up to order $N \sim 50$. As will be shown in detail in Sec. III, this provides a numerical tool that enables one to verify the results of the spectral theory of breather gases recently developed in ref. [23].

Fig. 1(a) shows the space-time evolution of a generic BG, i.e. a breather solution of Eq. (1) of order $N=50$ with random spectral charateristics. The amplitude of the plane wave background is unity $\left(q_{0}=\left|\psi_{0}\right|=1\right)$ and the 50 complex-valued eigenvalues $\lambda_{j}(j=1-50)$ parametrizing the BG are randomly distributed within some rectangular region of the upper complex plane, 
see Fig. 1(i). The parameters $t_{j}$ are fixed to zero $\left(t_{j}=0 \quad \forall j\right)$ and the randomness of the gas is achieved by uniformly distributing the $x_{j}$ in some interval centered around $x_{0}=0$. Note that the vertical line between 0 and $+i$ in Fig. 1(i) represents the so-called branchcut associated with the plane wave background in the IST formalism of the 1D-NLSE with NZBG, see e.g. [23, 34, 45, 46]. Fig. 1(a) reveals that the space-time dynamics of the generic BG synthesized in numerical simulations is highly complicated. In particular, breathers cannot be individualized due to their strong overlap and interaction. Note also that the maximum amplitude reached locally in space and time by the incoherent breather ensemble of Fig. 1(a) does not exceed $\sim 5.5$, which demonstrates that the multiple breathers are far from a synchronization state that would eventually produce isolated rogue waves of large amplitude [70, 71].

We emphasize that BGs shown in the space-time plots of Fig. 1 are not obtained from a numerical simulation of Eq. (1). Taking a BG generated at a given time $t_{0}$ using the Darboux method and using this wavefield as initial condition in a numerical simulation of Eq. (1), we observe that modulation instability quickly desintegrates the plane wave background by amplifying the numerical noise inherent to any pseudo-spectral (split-step like) method commonly used for the numerical integration of the 1D-NLSE. On the other hand space-time plots reported in Fig. 1 are obtained from a pure spectral (IST) construction based on the Darboux recursive method which has been implemented in computer simulations made with high numerical precision. Starting from an ensemble of $N$ complex eigenvalues $\lambda_{j}$ and $N$ coordinates $\left(x_{j}, t_{j}\right)$, the BG is synthesized at time $t$ using the Darboux machinary (Eqs. (5)-(10)). A 100 digits precision is typically necessary to synthesize a BG parametrized by an ensemble of $N \sim 50$ eigenvalues. The space-time plots shown in Fig. 1 are obtained by reiterating the same synthesis at different values of time $t$.

The central concept in the theory of SGs and BGs is the density of states (DOS) [72] which represents the distribution function $u(\lambda, x, t)$ in the spectral phase space. In the context of the 1D-NLSE (1) the DOS $u(\lambda, x, t)$, where $\lambda=\beta+i \gamma$, is defined such that $u d \beta d \gamma d x$ is the number of breather states with complex spectral parameter $\lambda \in[\beta, \beta+d \beta] \times[\gamma, \gamma+d \gamma]$ contained in a portion of $\mathrm{BG}$ within a spatial interval $[x, x+d x]$ at time $t$.

One-component BGs have been defined in ref. [23] as being characterized by a DOS in the form of the Dirac $\delta$ distribution, i.e. $u(\lambda)=w \delta\left(\lambda-\lambda_{0}\right)$ where $w>0$ represents the mass of the $\delta$ distribution which is centered around one specfic point $\lambda_{0}$ in the complex spectral plane. Fig. 1(b-d)(f-h) display the space-time evolutions together with the spectral portraits (Fig. 1(j-l)) typifying some one-component BGs of particular interest.

For the Kuznetsov-Ma BG (KM-BG), the spectral portait consists of the branchcut (associated with the plane wave background of unity amplitude) and a dense set of $N=50$ spectral points randomly placed in a small square region of width $\delta=10^{-3}$ centered around $\lambda_{0}=1.3 i$, as shown in Fig. 1(j). Fig. 1(b) shows that the KM-BG is a dense ensemble of individual KM breathers having all a zero velocity in the $(x, t)$-plane. Contrary to Fig. 1(a) each KM breather inside the BG can be individualized and it follows the same periodic time evolution where the time period is fully determined by $\Im\left(\lambda_{0}\right)$. The randomness in the one-component KM-BG can be seen from the random distance between individual KM breathers and their random initial phase, see Fig. 1(f).

The Akhmediev BG (AB-BG) is characterized by the same distribution of the spectrum $\lambda$ as the KM-BG except that the point $\lambda_{0}$ around which the multiple discrete eigenvalues are accumulated is now placed inside the branchcut associated with the plane wave background, see Fig. $1(\mathrm{k})$ where $\lambda_{0}=+0.8 i$. As a result, the AB-BG is more naturally characterized by the spectral flux density, the temporal counterpart of the DOS. As shown in Fig. 1(c), the AB-BG consists of a random series of individual ABs having identical spatial period, which is fully determined by $\Im\left(\lambda_{0}\right)$. Similarly to the KM-BG, the randomness in the one-component AB-BG can be seen from the random time separation between individual Akhmediev breathers and their random relative phases, see Fig. $1(\mathrm{~g})$.

It must be mentioned that the density (spatial or temporal) of the $\mathrm{AB}$ or $\mathrm{KM}$ breather gases cannot be made arbitrary large: there is a configuration termed "breather condensate" [23] corresponding to a critically dense breather gas, similar to a soliton condensate numerically realized in [19].

It is well known that the Peregrine breather can be obtained as the spatial and temporal infinite period limits of Akhmediev and Kuznetsov-Ma breathers respectively [66, 69]. In the spectral (IST) domain, the Peregrine breather is obtained by placing the eigenvalue parametrizing a first-order breather solution of Eq. (1) exactly at the endpoint $+i$ of the branchcut associated with the plane wave background of unit amplitude [34]. Following the same approach, the one-component Peregrine BG (P-BG) is obtained by accumulating a large number of discrete eigenvalues in a small area surrounding the endpoint of the branchcut, see Fig. 1(l). As shown in Fig. 1(d) and in Fig. 1(h), the P-BG represents a collection of individual and identical Peregrine breathers that are randomly positioned in space and time.

\section{INTERACTIONS IN BREATHER GASES: COMPARISON BETWEEN NUMERICAL EXPERIMENTS AND SPECTRAL THEORY}

The analytical theory of BGs has been introduced and developed in ref. [23]. It has been shown that spatially non-homogeneous BGs are described by a kinetic equation formed by a transport equation for the slowlyvarying DOS $u(\lambda, x, t)$ and the integral equation of state 


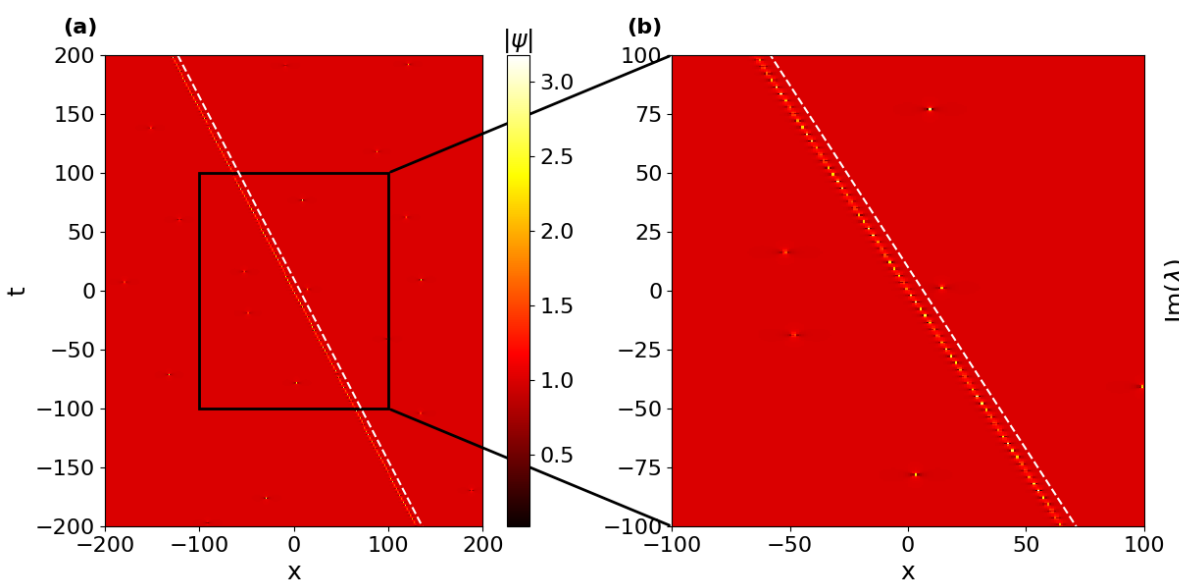

(c)

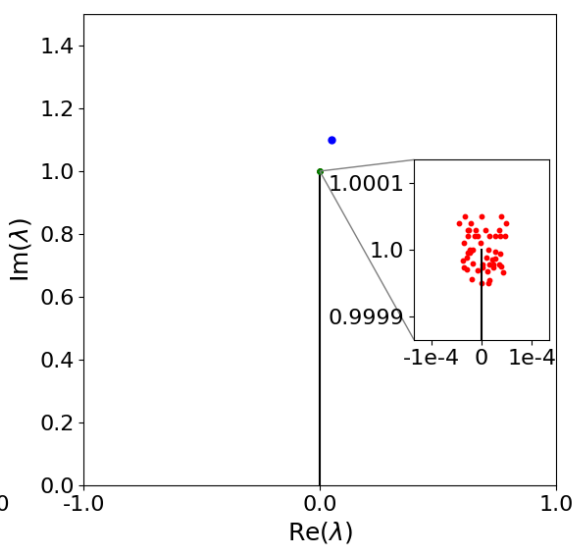

Figure 2. (a), (b) Propagation of a Tajiri-Watanabe breather with the spectral parameter $\eta^{[1]}=0.05+1.1 i$ inside a Peregrine BG. The space-time evolution shown in (b) represents an enlarged view of the one shown in (a). The white dashed line in (a) and (b) represents the trajectory of the "free" Tajiri-Watanabe breather propagating on a plane wave background with a group velocity given by Eq. (11). The plot shown in (c) represents the spectral portrait associated with the numerical results shown in (a), (b). The vertical line between 0 and $+i$ represents the branchcut associated with the plane wave background and the blue point is the discrete eigenvalue $\eta^{[1]}$ associated with the Tajiri-Watanabe breather propagating in the P-BG. The 50 spectral points characterizing the P-BG are densely placed around $+i$ and they are shown in the inset plotted in (c).

relating the gas' velocity to the DOS. In this Section, we show that some predictions of the spectral theory of BGs can be verified in simulations involving BGs that have been numerically synthesized using the methodology described in Sec. II B. In Sec. III A, we provide the key elements of spectral theory of BGs that are relevant for the comparison between theoretical and numerical results. In Sec. III B, we examine the collision between one trial soliton and various single-component BGs.

\section{A. Analytical results from the spectral theory of breather gases}

The nonlinear spectral theory of SGs and BGs for the focusing 1D-NLSE developed in ref. [23] provides a full set of equations characterizing the macroscopic spectral dynamics in a spatially nonhomogeneous BG.

An important result of the theory is the so-called equation of state which provides the mathematical expression of the modification of the mean velocity of a "tracer" breather due to its interaction with other breathers in the gas.

The group velocity (in the $(x, t)$-plane) of a first-order breather (TW) parametrized by the complex eigenvalue $\lambda \equiv \eta$ (we shall use in this section this latter notation for the spectral parameter to be consistent with notations of ref. [23] and previous works on the spectral kinetic theory) is given by

$$
s_{0}(\eta)=-2 \frac{\Im\left[\eta R_{0}(\eta)\right]}{\Im\left[R_{0}(\eta)\right]}
$$

where $R_{0}(z)=\sqrt{z^{2}-\delta_{0}^{2}}$ with $\delta_{0}$ the endpoint of the branchcut corresponding to the plane wave $\left(\delta_{0}=i\right.$ for the plane wave of unit amplitude considered in all the numerical simulations reported in this paper). It is not difficult to see that, if $\eta \in i \mathbb{R} \backslash[-i, i]$ (KM breather) then $s_{0}(\eta)=0$, while if $\eta \in(-i, i)(\mathrm{AB})$ then $s_{0}(\eta)= \pm \infty$ depending on the way the limit $\operatorname{Re}(\eta) \rightarrow 0$ in (11) is taken (either from the left or right side of the branch cut).

As shown in ref. [23], the equation of state of a BG reads

$$
s(\eta)=s_{0}(\eta)+\int_{\Lambda^{+}} \Delta(\eta, \mu)[s(\eta)-s(\mu)] u(\mu)|d \mu|
$$

where $\Lambda^{+}$is the 2D compact support of the DOS $u(\eta)$ (defined earlier in Section II B) located in the upper half plane $\mathbb{C}^{+}$of the complex spectral plane,

$$
\begin{aligned}
& \Delta(\eta, \mu)=\frac{1}{\Im\left[R_{0}(\eta)\right]}\left[\ln \left|\frac{\mu-\bar{\eta}}{\mu-\eta}\right|\right. \\
& \left.+\ln \left|\frac{R_{0}(\eta) R_{0}(\mu)+\eta \mu-\delta_{0}^{2}}{R_{0}(\bar{\eta}) R_{0}(\mu)+\bar{\eta} \mu-\delta_{0}^{2}}\right|\right] .
\end{aligned}
$$

The integral term in Eq. (12) describes the modification of the 'tracer' breather mean velocity in a gas due to its interaction with other breathers in the gas having a DOS specified by $u$. The spectral value $\eta$ in (12) can be taken outside $\Lambda^{+}$-in that case formula (12) describes the mean velocity of a "trial" or "test" TW breather with the eigenvalue $\eta$ propagating through a breather gas with DOS supported $\Lambda^{+}$.

The interaction kernel $\Delta(\eta, \mu)$ given by Eq. (13) describes the position shift arising in a two-breather interaction. We note that the two-breather interactions have been studied in [73], [49] using the IST, where different 


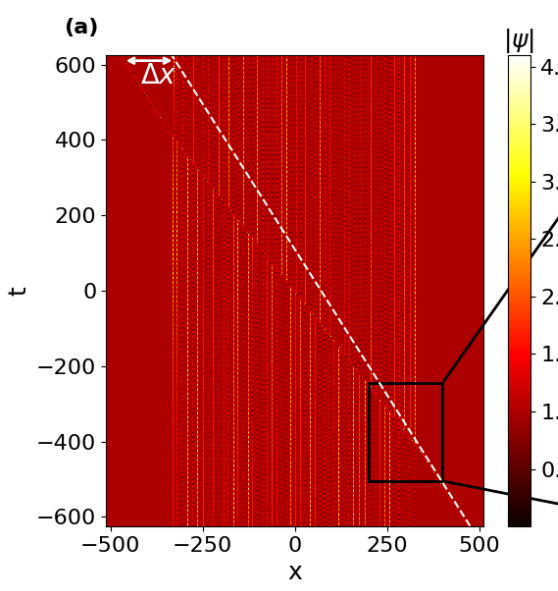

(b)

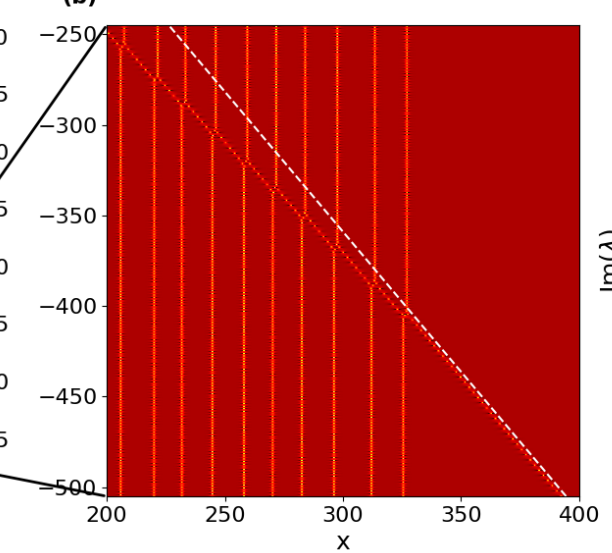

(c)

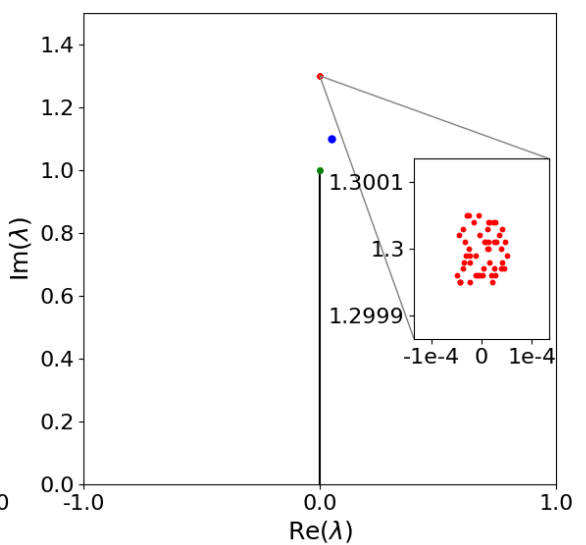

Figure 3. (a), (b) Propagation of a TW breather with the spectral parameter $\eta^{[1]}=0.05+1.1 i$ inside a Kuznetsov-Ma BG. The space-time evolution shown in (b) represents an enlarged view of the one shown in (a). The white dashed line in (a) and (b) represents the trajectory of the "free" TW breather propagating on a plane wave background with a group velocity given by Eq. (11). The plot shown in (c) represents the spectral portrait associated with the numerical results shown in (a), (b). The vertical line between 0 and $+i$ represents the branchcut associated with the plane wave background and the blue point is the discrete eigenvalue $\eta^{[1]}$ associated with the TW breather propagating in the KM-BG. The 50 spectral points characterizing the KM-BG are densely placed around $\eta^{[2]}=1.3 i$ and they are shown in the inset plotted in (c).

forms of the expressions for the position shift were obtained. In the Appendix we demonstrate the equivalence of the kernel $\Delta(\eta, \mu)$ given by (13) to the position shift formula obtained for two-breather collisions in previous works.

For a two-component breather gas, the DOS is a superposition of two Dirac delta-functions centered at the complex spectral points $\eta^{[j]}(j=1,2)$

$$
u(\eta)=\sum_{j=1}^{2} w^{[j]} \delta\left(\eta-\eta^{[j]}\right)
$$

where $w^{[j]}$ are the weights of the components. For the DOS specified by Eq. (14), Eq. (12) yields the following linear system for the gas' component velocities $s^{[j]} \equiv$ $s\left(\eta^{[j]}\right)(j=1,2)$

$$
\begin{aligned}
& s^{[1]}=s_{0}^{[1]}+\frac{\Delta_{1,2} w^{[2]}\left(s_{0}^{[1]}-s_{0}^{[2]}\right)}{1-\left(\Delta_{1,2} w^{[2]}+\Delta_{2,1} w^{[1]}\right)} \\
& s^{[2]}=s_{0}^{[2]}-\frac{\Delta_{2,1} w^{[1]}\left(s_{0}^{[1]}-s_{0}^{[2]}\right)}{1-\left(\Delta_{1,2} w^{[2]}+\Delta_{2,1} w^{[1]}\right)}
\end{aligned}
$$

where $s_{0}^{[j]} \equiv s_{0}\left(\eta^{[j]}\right)(j=1,2), \Delta_{j, k}=\Delta\left(\eta^{[j]}, \eta^{[k]}\right)$.

In the numerical simulations presented in Sec. III B, we will consider an even simpler situation where a single trial breather parametrized by the eigenvalue $\eta^{[1]}$ interacts with a one-component breather-gas having its spectral distribution centered in $\eta^{[2]}$. In such a limit $w^{[1]} \rightarrow 0$ and Eqs. (15) reduces to:

$$
\begin{aligned}
& s^{[1]}=\frac{s_{0}^{[1]}-\Delta_{1,2} w^{[2]} s_{0}^{[2]}}{1-\Delta_{1,2} w^{[2]}} . \\
& s^{[2]}=s_{0}^{[2]} .
\end{aligned}
$$

The validity of Eqs. (16) in the context of the 1DNLSE dynamics (1) will be verified for the P-BG, the KM-BG and the AB-BG in numerical simulations presented in Sec. IIIB. As a matter of fact, formula (16) can be obtained directly from equation (12) by setting $\eta=\eta^{[1]} \notin \Lambda^{+}$(the trial breather eigenvalue), and using $u(\mu)=w^{[2]} \delta\left(\mu-\eta^{[2]}\right), s\left(\eta_{2}\right)=s_{0}^{[2]}$ where $\eta^{[2]} \in \Lambda^{+}$.

\section{B. Interactions in one-component breather gases: Comparison between spectral theory and numerical simulations}

In the numerical simulations presented in this Section, a trial TW breather with the spectral parameter $\eta=\eta^{[1]}$ is propagated through various single-component BGs having their DOS defined by $u(\eta)=w^{[2]} \delta\left(\eta-\eta^{[2]}\right)$. We define spectral parameter $\eta^{[2]}$ as $\eta^{[2]}=\alpha i$ with $\alpha=1$ for the P-BG, $\alpha>1$ for the KM-BG, $\alpha<1$ for the ABBG. Similar to Fig. 1, the spectral portait of the considered BGs consists of the branchcut (associated with the plane wave background of unity amplitude) and a "cluster" of $N=50$ spectral points randomly placed in a small square region of width $\delta=10^{-4}$ centered around $\eta^{[2]}$. The spectral parameter $\eta^{[1]}$ is chosen in such a way that $\Re\left(\eta^{[1]}\right)>0$ which implies that the free trial TW breather has a negative group velocity in the $(x-t)$ plane, see Eq. (11).

\section{Interactions in the Peregrine breather gas}

Fig. 2 shows a trial Tajiri-Watanabe breather propagating through a P-BG. We observe that the trial 
breather passes through the P-BG without change in its group velocity. This confirms the theoretical result established in ref. [23] that the propagation of a trial TW breather through a P-BG is ballistic. This result can be understood at the qualitative level by the fact that the interaction cross section between the trial breather and the individual Peregrine breathers composing the gas is so weak that the propagation of the trial breather is unaffected by the P-BG.

\section{Interactions in the Kuznetsov-Ma breather gas}

Fig. 3 shows a trial TW breather propagating through a KM-BG. Contrary to Fig. 2, the multiple interactions between the trial breather and the KM breathers composing the KM-BG now significantly influences the propagation ot the trial breather, see Fig. 3(a) and 3(b) for a comparison between the trajectory of the free TajiriWatanabe breather (in white dashed lines) and the trajectory followed by the trial breather in the KM-BG. As shown in Fig. 3(b), the trial breather acquires a significant space shift each time that its trajectory intersects the trajectory of an individual KM breather composing the $\mathrm{BG}$. At the macroscopic scale, this produces a velocity change of the trial breather inside the KM-BG. This leads to a spatial shift $\Delta X$ in the position of the trial breather which is measurable when the trial breather emerges from the KM-BG, see Fig. 3(a).

For the KM-BG, Eq. (16) simplifies to

$$
s^{[1]}=\frac{s_{0}^{[1]}}{1-\Delta_{1,2} w^{[2]}}
$$

given that $s_{0}^{[2]}=0$. Eq. (17) clearly shows that the group velocity of the trial Tajiri-Watanabe breather is increased by a factor $1 /\left(1-\Delta_{1,2} w^{[2]}\right)$ due to the interaction with the KM-BG.

Note that the space shift $\Delta X$ acquired by the trial breather as a result of propagation inside the KM-BG simply represents the product of the number $N$ of interations (equivalently the number of breathers in the KM-BG) with the elementary space shift $\Delta_{1,2}$ induced by each interaction: $\Delta X=N \Delta_{1,2}$. This provides an alternative and straightforward way to check the validity of Eq. (17) which gives the group velocity of the trial breather inside the KM-BG.

A set of numerical simulations with different values of the spectral parameters $\eta^{[1]}$ and $\eta^{[2]}$ has been made to check the validity of the spectral theory. Different realizations of the KM-BG have been made and the value of $w^{[2]}$ is determined from numerical simulations as the ratio between the selected number $N$ of breathers in the gas over the spatial extension $L$ of the gas: $w^{[2]}=N / L$ As shown in Fig. 4, we observe full quantitative agreement between the numerical experiment and the predictions of the spectral theory.

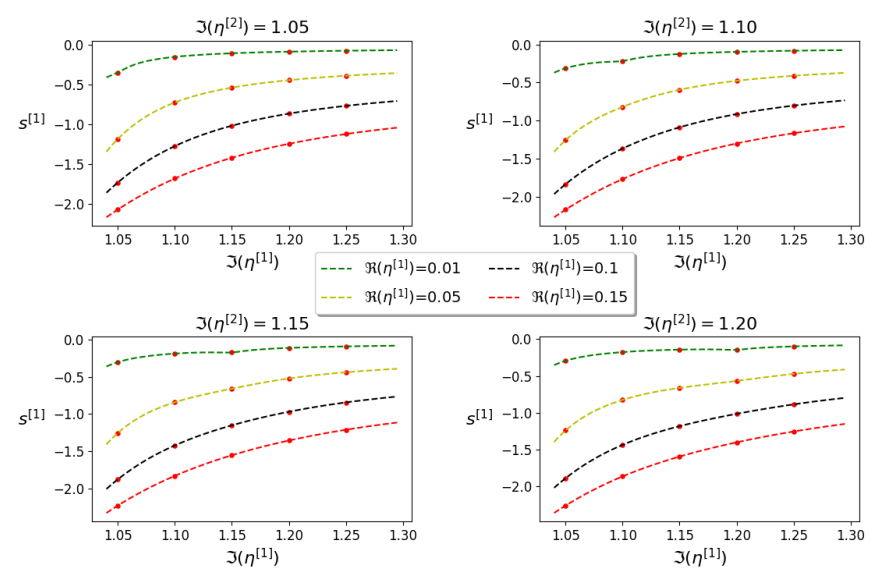

Figure 4. Quantitative verification of the spectral theory of BGs introduced in ref. [23]. Comparison between numerics (red dots) and theory (dashed lines) for the effective velocity $\left(s^{[1]}\right)$ of a trial breather $\left(\eta^{[1]}\right)$ propagating in a a KM-BG $\left(\eta^{[2]}\right)$.

\section{Interactions in the Akhmediev breather gas}

The case of $\mathrm{AB}-\mathrm{BG}$ is special and requires a separate consideration, particularly because it has not been considered in any detail in [23]. The AB is a "static" object, not localized in space, so it is not immediately obvious how to identify the key quantities $u(\eta)$ and $s(\eta)$ for the AB-BG. A single $\mathrm{AB}$ is a limiting case of the TW breather where the soliton eigenvalue $\eta^{[2]}$ is placed within the branch cut $[0, i]$ in the upper half plane, The $\mathrm{AB}-\mathrm{BG}$ is generally characterized by some distribution of soliton eigenvalues along the branch cut. Similar to the above consideration of KM-BG, we consider the AB-BG with soliton eigenvalues clustered around a given spectral point $\eta^{[2]}$ (and c.c.) to mimic a one-component gas.

As we have already mentioned in Section III A the formula (11) for the group velocity of the TW breather implies $|s(\eta)| \rightarrow \infty$ as $\eta \rightarrow \eta^{[2]}$, which is consistent with the delocalized nature of the $\mathrm{AB}$. On the other hand, it can be shown using the results of ref. [23], that in the AB-BG limit the DOS $u(\eta) \rightarrow 0$ while the spectral flux function $v(\eta)=s(\eta) u(\eta)=\mathcal{O}(1)$. This motivates the following alternative form of the equation of state (12):

$$
s(\eta)=s_{0}(\eta)+\int_{\Lambda^{+}} \Delta(\eta, \mu)\left[\frac{s(\eta)}{s(\mu)}-1\right] v(\mu)|d \mu|,
$$

which is more suitable for the characterization of the ABBG interactions. Equation (18) was obtained from (12) by substituting $u(\eta)=\frac{v(\eta)}{s(\eta)}$. Assuming $\Lambda^{+}$to be a narrow region surrounding the branch cut $[0, i]$ and using $|s(\mu)| \gg 1$ for $\mu \in \Lambda^{+}$equation (18) to leading order becomes

$$
s(\eta)=s_{0}(\eta)-\int_{\Lambda^{+}} \Delta(\eta, \mu) v(\mu)|d \mu| .
$$




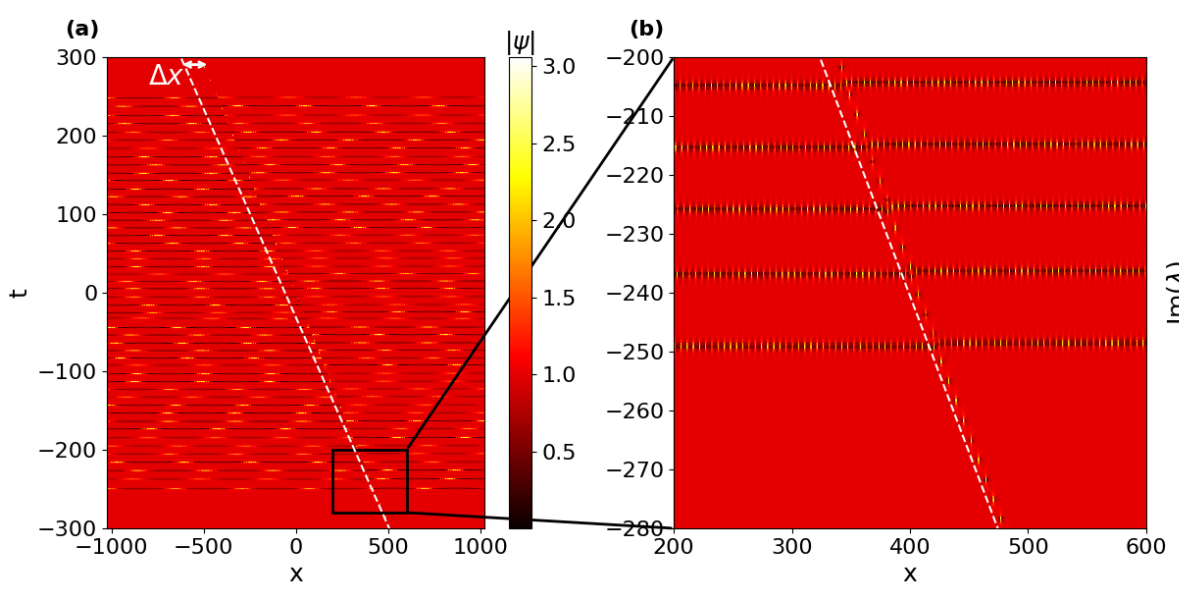

(c)

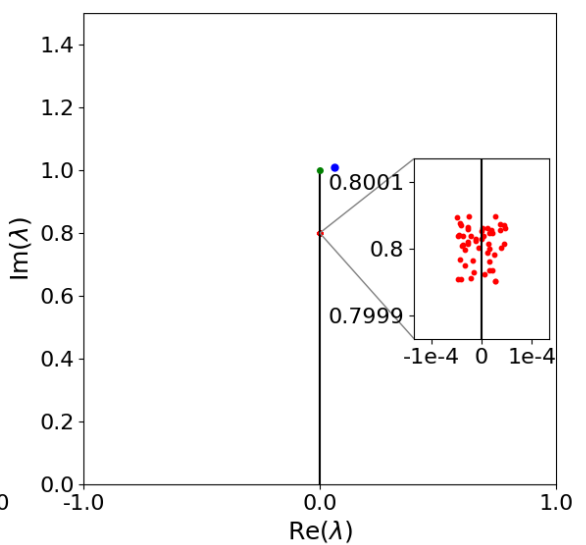

Figure 5. (a), (b) Propagation of a TW breather with the spectral parameter $\eta_{1}=0.06+1.01 i$ inside a Akhmediev BG. The space-time evolution shown in (b) represents an enlarged view of the one shown in (a). The white dashed line in (a) and (b) represents the trajectory of the "free" TW breather propagating on a plane wave background with a group velocity given by Eq. (11). The plot shown in (c) represents the spectral portrait associated with the numerical results shown in (a), (b). The vertical line between 0 and $+i$ represents the branch cut associated with the plane wave background and the blue point is the discrete eigenvalue $\eta_{1}$ associated with the TW breather propagating in the AB-BG. The 50 spectral points characterizing the KM-BG are densely placed around $\eta^{[2]}=0.8 i$ and they are shown in the inset plotted in (c).

Equation (19) describes the modification of the velocity of the TW breather with eigenvalue $\eta$ propagating through the AB-BG characterized by the spectral flux density $v(\mu)$.

An important property of $\Delta(\eta, \mu)$ given by (13) is that

$$
\Delta(\eta, \mu)+\Delta(\eta,-\bar{\mu})=0 \quad \text { when } \quad \mu \in[0, i],
$$

that is, when $\mu$ is on the branch cut $[0, i]$. The second variable $\eta$ can take any value in the upper half-plane. Equation (20) implies that $\Delta(\eta, \mu)$ takes opposite values on the opposite sides of the branchcut.

It can further be shown that in the case of a breather gas, whose spectral support $\Lambda^{+}$is symmetric with respect to the branch cut $[0, i]$, the function $v(\eta)$ also takes opposite values on the opposite sides of $[0, i]$. Thus the speed of the AB-BG $s(\eta)$ from (18) does not depend on which side of the upper part of the branch cut $[0, i]$ the domain $\Lambda^{+}$or its parts are situated.

Let us now consider a one-component AB-BG with the spectral flux $v(\eta)=w^{t} \delta\left(\eta-\eta^{[2]}\right)$, where $\eta^{[2]} \in[0, i]$ and $w^{t}$ is a real constant weight. As a result, equation (19) assumes a simple form

$$
s(\eta)=s_{0}(\eta)-w^{t} \Delta\left(\eta, \eta^{[2]}\right),
$$

We note that the sign of $w^{t}$, as was explained above, depends on the side of $[0, i]$ but the sign of the product $w^{t} \Delta$ does not. Hence we have the general result $s(\eta)-$ $s_{0}(\eta)<0$ for the propagation of a trial breather through a AB-BG.

We note that formula (21) can be obtained directly from the basic result (16) by using $w^{[2]} \rightarrow 0$ and introducing $w^{[2]} s_{0}^{[2]} \equiv w^{t}$. This simple formal consideration, however, does not provide the important information about the sign of $w^{t} \Delta$.
Fig. 5 shows a trial TW breather propagating through a AB-BG. Similar to Fig. 3, the propagation of the trial breather is significantly influenced by the the multiple interactions with the $\mathrm{AB}$ breathers composing the $\mathrm{AB}$ BG, see Fig. 5(a) and 5(b). One can see that, in contrast to the interaction of the trial TW breather with the KM-BG, the group velocity of the trial TW breather is reduced in the interaction with the $\mathrm{AB}-\mathrm{BG}$, in agreement with Eq. (21). Indeed, the space shifts observed in Fig. 3(a) and in Fig. 5(a) have opposite signs.

Similar to the KM-BG interactions, a set of numerical simulations with different values of the spectral parameters $\eta^{[1]}$ and $\eta^{[2]}$ has been made to check the validity of equation (21). Different realizations of the AB-BG have been produced and the value of $w^{t}$ was determined from numerical simulations as the ratio between the selected number $N$ of $\mathrm{AB}$ in the gas over the temporal extension $T$ of the gas: $w^{t}=N / T$ As shown in Fig. 6, we observe full quantitative agreement between the numerical experiment and the predictions of the spectral theory.

\section{CONCLUSIONS}

We have developed a numerical algorithm of the IST spectral synthesis of breather gases for the focusing 1DNLS equation. The algorithm is based on the recursive Darboux transform scheme realized in high precision arithmetics. Using this algorithm we have synthesized numerically three types of "prototypical" breather gases: the Akhmediev, Kuznetsov-Ma and Peregrine gas.

Using the developed spectral algorithm, the interaction properties of breather gases, predicted by the kinetic theory of ref. [23] have been tested by propagating through 

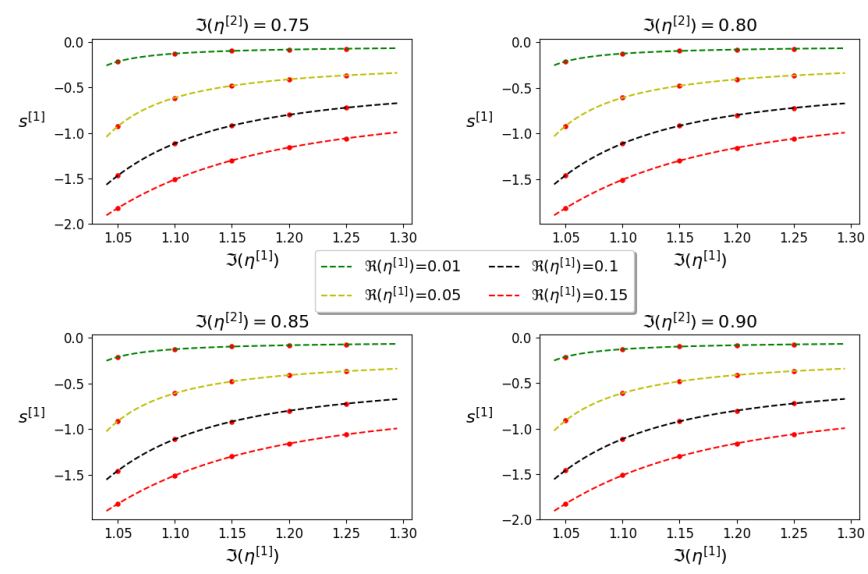

Figure 6. Comparison between numerics (red dots) and theory (dashed lines) for the effective velocity $\left(s^{[1]}\right)$ of a trial TW breather $\left(\eta^{[1]}\right)$ propagating in a a AB-BG $\left(\eta^{[2]}\right)$.

them a 'trial' generic TW breather whose effective velocity is strongly affected by the interaction with the gas. In all cases the theoretically predicted effective mean velocity of the trial breather propagating through a breather gas demonstrates excellent agreement with the results of the numerical simulations. The verification of the theory, despite the inevitable effects of modulational instability present in the 1D-NLSE dynamics, has been made possible due to the whole numerical algorithm being based on the spectral construction rather than direct simulations of the 1D-NLSE equation.

The quantitative verification of the kinetic theory of breather gases undertaken in this paper is an important step towards a better understanding of this type of a turbulent motion in integrable systems. We also believe that the ability to synthesize numerically BGs represents a step of importance towards the controlled laboratory generation of BGs, possibly following an approach similar to the one recently reported for hydrodynamic SGs [18]. Finally the possibility to generate numerically breather solutions of order $N \gtrsim 10$ paves the way for further works devoted to the investigation of the properties of localization in space and time of breather solutions of the 1DNLSE of very high order [37, 49, 71].

\section{ACKNOWLEDGMENTS}

This work has been partially supported by the Agence Nationale de la Recherche through the I-SITE ULNE (ANR-16-IDEX-0004), the LABEX CEMPI (ANR-11LABX-0007) and the Equipex Flux (ANR-11-EQPX-
0017), as well as by the Ministry of Higher Education and Research, Hauts de France council and European Regional Development Fund (ERDF) through the CPER project Photonics for Society (P4S), EPSRC grant (UK) EP/R00515X/2 (GE), NSF (USA) grant DMS 2009647 (AT) and Dstl (UK) grant DSTLX-1000116851 (GR, GE, SR). GE, AT and GR thank the PhLAM laboratory at the University of Lille for hospitality and partial financial support.

\section{APPENDIX: POSITION SHIFT IN TWO-BREATHER INTERACTIONS}

The two-breather interactions have been studied in refs. [73], [49] where the expressions for the phase and position shifts in the interaction of two Tajiri-Watanabe breathers have been derived using the IST analysis. In Section III A of this paper the interaction kernel in the equation of state (12) for breather gas has been obtained in the form (13). The natural interpretation of this interaction kernel, consistent with the previously studied cases of KdV and NLS soliton gases, is the position shift in a two-breather collision. However, the equivalence between formula (13) and the expressions from [73], [49] is far from being obvious. Here we establish this equivalence enabling one to extend the phenomenological interpretation of soliton gas kinetics [22] to breather gases.

We consider the position shift expression from [73]

$$
\Delta \bar{\xi}_{2}=-\ln \left(\xi_{0}\right) /\left(c_{-, 2} \cos \alpha_{2}\right)=\Delta\left(\lambda_{2}, \lambda_{1}\right),
$$

where

$$
\xi_{0}=\frac{d_{+}-2\left(\cos \left(\alpha_{1}-\alpha_{2}\right)+c_{-, 1} c_{-, 2}\right) \cos \left(\alpha_{1}-\alpha_{2}\right)}{d_{+}-2\left(\cos \left(\alpha_{1}+\alpha_{2}\right)-c_{-, 1} c_{-, 2}\right) \cos \left(\alpha_{1}+\alpha_{2}\right)}
$$

with

$$
\begin{aligned}
& c_{ \pm, j}=z_{j} \pm q_{0}^{2} / z_{j} \quad \lambda_{j}=\left(\zeta_{j}-q_{0}^{2} / \zeta_{j}\right) / 2 \\
& d_{ \pm, j}=z_{j}^{2} \pm q_{0}^{4} / z_{j}^{2} \quad q_{0}=-i \delta_{0} \\
& d_{+}=d_{+, 1}+d_{+, 2} \quad R_{0}\left(\lambda_{j}\right)=\left(\zeta_{j}+q_{0}^{2} / \zeta_{j}\right) / 2 \\
& \zeta_{j}=R_{0}\left(\lambda_{j}\right)+\lambda_{j}=i z_{j} e^{i \alpha_{j}} .
\end{aligned}
$$

One can verify that substituting (24) in (13) and invoking the identities

$$
\begin{aligned}
& \left|\lambda_{i}\right|^{2}=\left(d_{+, i}+2 q_{0}^{2} \cos \alpha_{i}\right) / 4 \\
& d_{+}=\left(z_{1} z_{2}+\frac{q_{0}^{4}}{z_{1} z_{2}}\right)\left(\frac{z_{1}}{z_{2}}+\frac{z_{2}}{z_{1}}\right) \\
& \left(\cos 2 \alpha_{1}+\cos 2 \alpha_{2}\right) / 2=\cos \left(\alpha_{1}+\alpha_{2}\right) \cos \left(\alpha_{1}-\alpha_{2}\right)
\end{aligned}
$$

yields the phase shift expression (22).
[1] Sergei Petrovich Novikov, S V Manakov, Lev Petrovich Pitaevskii, and Vladimir E Zakharov,
Theory of solitons: the inverse scattering method 
(Springer Science Business Media, 1984).

[2] Vladimir E Zakharov, "Turbulence in integrable systems," Stud. Appl. Math. 122, 219-234 (2009).

[3] Michelle D. Maiden, Dalton V. Anderson, Nevil A. Franco, Gennady A. El, and Mark A. Hoefer, "Solitonic dispersive hydrodynamics: Theory and observation," Physical Review Letters 120, 144101 (2018).

[4] G. A. El and M. A. Hoefer, "Dispersive shock waves and modulation theory," Physica D: Nonlinear Phenomena Dispersive Hydrodynamics, 333, 11-65 (2016).

[5] A A Gelash and V E Zakharov, "Superregular solitonic solutions: a novel scenario for the nonlinear stage of modulation instability," Nonlinearity 27, R1 (2014).

[6] B. Kibler, A. Chabchoub, A. Gelash, N. Akhmediev, and V. E. Zakharov, "Superregular breathers in optics and hydrodynamics: Omnipresent modulation instability beyond simple periodicity," Phys. Rev. X 5, 041026 (2015).

[7] Gang Xu, Andrey Gelash, Amin Chabchoub, Vladimir Zakharov, and Bertrand Kibler, "Breather wave molecules," Phys. Rev. Lett. 122, 084101 (2019).

[8] J. M. Soto-Crespo, N. Devine, and N. Akhmediev, "Integrable turbulence and rogue waves: Breathers or solitons?" Phys. Rev. Lett. 116, 103901 (2016).

[9] N. Akhmediev, J. M. Soto-Crespo, and N. Devine, "Breather turbulence versus soliton turbulence: Rogue waves, probability density functions, and spectral features," Phys. Rev. E 94, 022212 (2016).

[10] Stéphane Randoux, Pierre Walczak, Miguel Onorato, and Pierre Suret, "Intermittency in integrable turbulence," Phys. Rev. Lett. 113, 113902 (2014).

[11] Pierre Walczak, Stéphane Randoux, and Pierre Suret, "Optical rogue waves in integrable turbulence," Phys. Rev. Lett. 114, 143903 (2015).

[12] Pierre Suret, Rebecca El Koussaifi, Alexey Tikan, Clément Evain, Stéphane Randoux, Christophe Szwaj, and Serge Bielawski, "Single-shot observation of optical rogue waves in integrable turbulence using time microscopy," Nature Communications 7, 13136 (2016).

[13] Guillaume Michel, Félicien Bonnefoy, Guillaume Ducrozet, Gaurav Prabhudesai, Annette Cazaubiel, Francois Copie, Alexey Tikan, Pierre Suret, Stéphane Randoux, and Eric Falcon, "Emergence of peregrine solitons in integrable turbulence of deep water gravity waves," Phys. Rev. Fluids 5, 082801 (2020).

[14] Andrea Costa, Alfred R. Osborne, Donald T. Resio, Silvia Alessio, Elisabetta Chrivì, Enrica Saggese, Katinka Bellomo, and Chuck E. Long, "Soliton turbulence in shallow water ocean surface waves," Phys. Rev. Lett. 113, 108501 (2014).

[15] J. Wang, Q. W. Ma, S. Yan, and A. Chabchoub, "Breather rogue waves in random seas," Phys. Rev. Applied 9, 014016 (2018).

[16] Alfred R. Osborne, Donald T. Resio, Andrea Costa, Sonia Ponce de Leòn, and Elisabetta Chirivì, "Highly nonlinear wind waves in Currituck Sound: dense breather turbulence in random ocean waves," Ocean Dynamics 69, 187-219 (2019).

[17] Ivan Redor, Eric Barthélemy, Hervé Michallet, Miguel Onorato, and Nicolas Mordant, "Experimental evidence of a hydrodynamic soliton gas," Phys. Rev. Lett. 122, 214502 (2019).

[18] Pierre Suret, Alexey Tikan, Félicien Bonnefoy, Fran çois Copie, Guillaume Ducrozet, Andrey Gelash, Gaurav Prabhudesai, Guillaume Michel, Annette Cazaubiel, Eric
Falcon, Gennady El, and Stéphane Randoux, "Nonlinear spectral synthesis of soliton gas in deep-water surface gravity waves," Phys. Rev. Lett. 125, 264101 (2020).

[19] Andrey Gelash, Dmitry Agafontsev, Vladimir Zakharov, Gennady El, Stéphane Randoux, and Pierre Suret, "Bound state soliton gas dynamics underlying the spontaneous modulational instability," Phys. Rev. Lett. 123, $234102(2019)$.

[20] V. E. Zakharov, "Kinetic equation for solitons," Sov. Phys.-JETP 33, 538-541 (1971).

[21] G.A. El, "The thermodynamic limit of the Whitham equations," Physics Letters A 311, 374-383 (2003).

[22] G. A. El and A. M. Kamchatnov, "Kinetic equation for a dense soliton gas," Phys. Rev. Lett. 95, 204101 (2005).

[23] Gennady El and Alexander Tovbis, "Spectral theory of soliton and breather gases for the focusing nonlinear schrödinger equation," Phys. Rev. E 101, 052207 (2020).

[24] Nail Akhmediev, Adrian Ankiewicz, and J. M. SotoCrespo, "Rogue waves and rational solutions of the nonlinear schrödinger equation," Phys. Rev. E 80, 026601 (2009).

[25] N. Akhmediev, A. Ankiewicz, and M. Taki, "Waves that appear from nowhere and disappear without a trace," Phys. Lett. A 373, 675 - 678 (2009).

[26] David J. Kedziora, Adrian Ankiewicz, and Nail Akhmediev, "Second-order nonlinear schrödinger equation breather solutions in the degenerate and rogue wave limits," Phys. Rev. E 85, 066601 (2012).

[27] G. Genty, C.M. de Sterke, O. Bang, F. Dias, N. Akhmediev, and J.M. Dudley, "Collisions and turbulence in optical rogue wave formation," Physics Letters A 374, $989-996$ (2010).

[28] John M. Dudley, Goëry Genty, Arnaud Mussot, Amin Chabchoub, and Frédéric Dias, "Rogue waves and analogies in optics and oceanography," Nature Reviews Physics 1, 675 (2019).

[29] E. A. Kuznetsov, "Solitons in a parametrically unstable plasma," Akademiia Nauk SSSR Doklady 236, 575-577 (1977).

[30] D. H. Peregrine, "Water waves, nonlinear schrödinger equations and their solutions," The Journal of the Australian Mathematical Society. Series B. Applied Mathematics 25, 16-43 (1983).

[31] N. N. Akhmediev and V. I. Korneev, "Modulation instability and periodic solutions of the nonlinear schrödinger equation," Theor. Math. Phys. 69, 1089-1093 (1986).

[32] Bertrand Kibler, Julien Fatome, Christophe Finot, Guy Millot, Frédéric Dias, Goëry Genty, Nail Akhmediev, and John M Dudley, "The peregrine soliton in nonlinear fibre optics," Nature Physics 6, 790-795 (2010).

[33] A. Chabchoub, N. P. Hoffmann, and N. Akhmediev, "Rogue wave observation in a water wave tank," Phys. Rev. Lett. 106, 204502 (2011).

[34] Stéphane Randoux, Pierre Suret, and Gennady El, "Inverse scattering transform analysis of rogue waves using local periodization procedure," Scientific reports 6, 29238 (2016).

[35] Masayoshi Tajiri and Yosuke Watanabe, "Breather solutions to the focusing nonlinear schrödinger equation," Phys. Rev. E 57, 3510-3519 (1998).

[36] N.N. Akhmediev, V.I. Korneev, and N.V. Mitskevich, "N-modulation signals in a single-mode optical waveguide under nonlinear conditions," Sov. Phys.-JETP 67, 89-95 (1988). 
[37] David J. Kedziora, Adrian Ankiewicz, and Nail Akhmediev, "Classifying the hierarchy of nonlinear-schrödingerequation rogue-wave solutions," Phys. Rev. E 88, 013207 (2013).

[38] A. A. Gelash and D. S. Agafontsev, "Strongly interacting soliton gas and formation of rogue waves," Phys. Rev. E 98, 042210 (2018).

[39] V. E. Zakharov and A. B. Shabat, "Exact theory of two-dimensional self-focusing and one-dimensional selfmodulation of waves in nonlinear media," Sov. Phys.JETP 34, 62-69 (1972).

[40] J. Yang, Nonlinear Waves in Integrable and Non-integrable Sf508je Mathematical Modeling and Computation (Society for Industrial and Applied Mathematics, 2010).

[41] S. Wahls and H. V. Poor, "Fast numerical nonlinear fourier transforms," IEEE Transactions on Information Theory 61, 6957-6974 (2015).

[42] Son Thai Le, Vahid Aref, and Henning Buelow, "Nonlinear signal multiplexing for communication beyond the kerr nonlinearity limit," Nat. Photon. 11, 570 (2017).

[43] Sergei K. Turitsyn, Jaroslaw E. Prilepsky, Son Thai Le, Sander Wahls, Leonid L. Frumin, Morteza Kamalian, and Stanislav A. Derevyanko, "Nonlinear fourier transform for optical data processing and transmission: advances and perspectives," Optica 4, 307-322 (2017).

[44] Yu-Hao Sun, "Soliton synchronization in the focusing nonlinear schrödinger equation," Phys. Rev. E 93, 052222 (2016).

[45] Gino Biondini and Gregor Kovačič, "Inverse scattering transform for the focusing nonlinear schrödinger equation with nonzero boundary conditions," J. Math. Phys. 55, 031506 (2014).

[46] Gino Biondini and Emily Fagerstrom, "The integrable nature of modulational instability," SIAM J. Appl. Math. 75, 136-163 (2015).

[47] Yan-Chow Ma, "The perturbed plane-wave solutions of the cubic schrödinger equation," Studies in Applied Mathematics 60, 43-58 (1979).

[48] V. E. Zakharov and A. A. Gelash, "Nonlinear stage of modulation instability," Phys. Rev. Lett. 111, 054101 (2013).

[49] A. A. Gelash, "Formation of rogue waves from a locally perturbed condensate," Phys. Rev. E 97, 022208 (2018).

[50] A. Chabchoub, N. Hoffmann, M. Onorato, and N. Akhmediev, "Super rogue waves: Observation of a higher-order breather in water waves," Phys. Rev. X 2, 011015 (2012).

[51] A. Chabchoub, N. Hoffmann, M. Onorato, A. Slunyaev, A. Sergeeva, E. Pelinovsky, and N. Akhmediev, "Observation of a hierarchy of up to fifth-order rogue waves in a water tank," Phys. Rev. E 86, 056601 (2012).

[52] B. Frisquet, B. Kibler, and G. Millot, "Collision of akhmediev breathers in nonlinear fiber optics," Phys. Rev. X 3, 041032 (2013).

[53] Bertrand Kibler, Julien Fatome, Christophe Finot, Guy Millot, Goëry Genty, Benjamin Wetzel, N Akhmediev, Frédéric Dias, and John M Dudley, "Observation of kuznetsov-ma soliton dynamics in optical fibre," Sci. Rep. 2 (2012).

[54] J. M. Dudley, G. Genty, F. Dias, B. Kibler, and N. Akhmediev, "Modulation instability, akhmediev breathers and continuous wave supercontinuum generation," Opt. Express 17, 21497-21508 (2009).

[55] Jan-Willem Goossens, Hartmut Hafermann, and Yves
Jaouën, "Experimental realization of fermi-pasta-ulamtsingou recurrence in a long-haul optical fiber transmission system," Scientific reports 9, 18467 (2019).

[56] Giovanni Dematteis, Tobias Grafke, Miguel Onorato, and Eric Vanden-Eijnden, "Experimental evidence of hydrodynamic instantons: The universal route to rogue waves," Phys. Rev. X 9, 041057 (2019).

[57] P. Dubard, P. Gaillard, C. Klein, and V.B. Matveev, "On multi-rogue wave solutions of the nls equation and positon solutions of the kdv equation," The European Physical Journal Special Topics 185, 247-258 (2010).

erRs,Dubard and V. B. Matveev, "Multi-rogue waves solutions to the focusing nls equation and the $\mathrm{kp}$-i equation," Natural Hazards and Earth System Sciences 11, 667-672 (2011).

[59] Pierre Gaillard, "Families of quasi-rational solutions of the NLS equation and multi-rogue waves," Journal of Physics A: Mathematical and Theoretical 44, 435204 (2011).

[60] Adrian Ankiewicz, David J. Kedziora, and Nail Akhmediev, "Rogue wave triplets," Physics Letters A 375, 2782 - 2785 (2011).

[61] Yasuhiro Ohta and Jianke Yang, "General high-order rogue waves and their dynamics in the nonlinear schrödinger equation," Proceedings of the Royal Society A: Mathematical, Physical and Engineering Sciences 468, 1716-1740 (2012).

[62] J. S. He, H. R. Zhang, L. H. Wang, K. Porsezian, and A. S. Fokas, "Generating mechanism for higher-order rogue waves," Phys. Rev. E 87, 052914 (2013).

[63] Wen-Rong Sun, Lei Liu, and P. G. Kevrekidis, "Rogue waves of ultra-high peak amplitude: A mechanism for reaching up to thousand times the background level," (2020), arXiv:2007.00393 [nlin.PS].

[64] Chaohao $\mathrm{Gu}$, Hesheng $\mathrm{Hu}, \quad$ and Zixiang Zhou, " $1+1$ dimensional integrable systems," in Darboux Transformations in Integrable Systems: Theory and their $A$ (Springer Netherlands, Dordrecht, 2005) pp. 1-64.

[65] N. N. Akhmediev and N. V. Mitzkevich, "Extremely high degree of n-soliton pulse compression in an optical fiber," IEEE Journal of Quantum Electronics 27, 849857 (1991).

[66] N. Akhmediev, J.M. Soto-Crespo, and A. Ankiewicz, "Extreme waves that appear from nowhere: On the nature of rogue waves," Physics Letters A 373, 2137 - 2145 (2009).

[67] Boling Guo, Liming Ling, and Q. P. Liu, "Nonlinear schrödinger equation: Generalized darboux transformation and rogue wave solutions," Phys. Rev. E 85, 026607 (2012).

[68] F. J. García-Gómez and V. Aref, "Statistics of the nonlinear discrete spectrum of a noisy pulse," Journal of Lightwave Technology 37, 3563-3570 (2019).

[69] David J. Kedziora, Adrian Ankiewicz, and Nail Akhmediev, "Circular rogue wave clusters," Phys. Rev. E 84, 056611 (2011).

[70] Bertola M. and Tovbis A., "Maximal amplitudes of finitegap solutions for the focusing nonlinear schrödinger equation," Communications in Mathematical Physics 354, 525 - 547 (2017).

[71] Bo Yang and Jianke Yang, "Rogue wave patterns in the nonlinear schrödinger equation," (2021), arXiv:2101.00383 [nlin.SI].

[72] IM Lifshits, SA Gredeskul, and LA Pastur, 
Introduction to the theory of disordered systems (Wiley, 1988).

[73] Sitai Li and Gino Biondini, "Soliton interactions and degenerate soliton complexes for the focusing nonlinear schrödinger equation with nonzero background," Eur. Phys. J. Plus 133 (2018). 